\section{Síndrome hepatopulmonar en pediatría. Serie de casos y revisión de la literatura}

López-Birlain $\mathrm{L}^{1}$, Cuevas-Schacht $\mathrm{FJ}^{2}$, Gutiérrez-Morales $\mathrm{G}^{3}$, Alva-Chaire $\mathrm{AC}^{3}$

\begin{abstract}
Resumen
El síndrome hepatopulmonar es una entidad poco frecuente en la población pediátrica, con una incidencia desconocida en México. Su diagnóstico supone un verdadero reto para el clínico, debido a la amplia gama de causas de hipoxemia en los pacientes con hepatopatías crónicas.
\end{abstract}

Se presentan dos casos de pacientes diagnosticados con síndrome hepatopulmonar mostrando la variabilidad en la presentación y evolución del mismo; una paciente con hepatopatía crónica secundaria a una enfermedad autoinmune (lupus eritematoso sistémico) con una evolución tórpida, y otra paciente con diagnóstico de hepatopatía, posterior al inicio de la sintomatología respiratoria. En ambos casos el tratamiento ideal consistiría en trasplante hepático; sin embargo, éste no pudo realizarse debido a la dificultad para el acceso al mismo en nuestro país.

PALABRAS CLAVE: hepatopulmonar, hipoxemia, hepatopatía.

\section{Pediatric hepatopulmonary syndrome. Case report and a literature review.}

López-Birlain $\mathrm{L}^{1}$, Cuevas-Schacht FJ $\mathrm{J}^{2}$, Gutiérrez-Morales $\mathrm{G}^{3}$, Alva-Chaire $\mathrm{AC}^{3}$

\begin{abstract}
The hepatopulmonary syndrome is an infrequent disease in pediatric population, with an unknown incidence in Mexico. The diagnosis is a challenge for the clinician due to the presence of many other causes of hypoxemia in children with chronic liver disease.

Two patients with Hepatopulmonary Syndrome are presented, showing the variability in both cases and outcome of the disease. First a patient with chronic liver disease secondary to an autoimmune disease (Systemic Lupus Erythematosus) with a torpid evolution, then a patient in which the diagnosis of chronic liver disease was made after the onset of the respiratory symptoms. In both cases the ideal treatment should be hepatic transplantation, however it could not be done because of the low accessibility to this surgical procedure in our country.
\end{abstract}

KEYWORDS: hepatopulmonary; hypoxemia; liver disease
${ }^{1}$ Residente de la subespecialidad en Neumología Pediátrica.

2Jefe del Departamento de Neumología y Cirugía de Tórax.

${ }^{3}$ Médico adscrito al Departamento de Neumología y Cirugía de Tórax.

Instituto Nacional de Pediatría.

Recibido: 28 de julio del 2016

Aceptado: 13 de diciembre del 2016

\section{Correspondencia}

Luis López-Birlain

luislopb@hotmail.com

Este artículo debe citarse como López-Birlain L, Cuevas-Schacht FJ, Gutiérrez-Morales G, Alva-Chaire AC. Síndrome hepatopulmonar en pediatría. Serie de casos y revisión de la literatura. Acta Pediatr Mex. 2017;38(6):371-377. 


\section{INTRODUCCIÓN}

El síndrome hepatopulmonar es un trastorno respiratorio secundario a daño hepático por distintas etiologías. La sospecha clínica y la acuciosidad en la realización de la historia clínica e interpretación de estudios de laboratorio y gabinete son fundamentales para su diagnóstico. A continuación se presentan dos casos de pacientes diagnosticados con síndrome hepatopulmonar y una revisión de literatura actual y relevante del tema.

\section{Caso 1}

Niña de 13 años de edad con 1 año de evolución con aumento de volumen abdominal, ictericia en escleras, astenia, adinamia y disnea de esfuerzo progresiva. Un mes previo a su valoración inicial, presenta evacuaciones disminuidas de consistencia de más de 20 días de evolución, inversión del ciclo sueño-vigilia y red venosa colateral en abdomen y extremidades. Se identifica hepatoesplenomegalia (hígado $8 \times 10 \times 14 \mathrm{~cm}$ por debajo del reborde costal derecho, polo esplénico $5 \mathrm{~cm}$ por debajo del reborde costal izquierdo), taquicardia, taquipnea y oximetría de pulso de $84 \%$. Se descarta insuficiencia cardiaca y mediante ecocardiograma transtorácico (EcoTT) se calcula una presión sistólica de la arteria pulmonar (PSAP) de 10 a 15mmHg. En los exámenes de laboratorio destaca únicamente la elevación de enzimas hepáticas. En ultrasonido hepático se reporta corto circuito espleno-cava.

La valoración por Neumología documenta clínicamente disnea de moderados a pequeños esfuerzos, cianosis ungueal, taquipnea y oximetría de pulso de $76 \%$, platipnea y ortodesoxia. La radiografía de tórax demuestra infiltrado intersticial reticular bilateral (Figura 1). Ante la presencia de hepatopatía crónica secundaria a lupus eritematoso sistémico y síndrome hemofagocítico primario, acompañado de afección

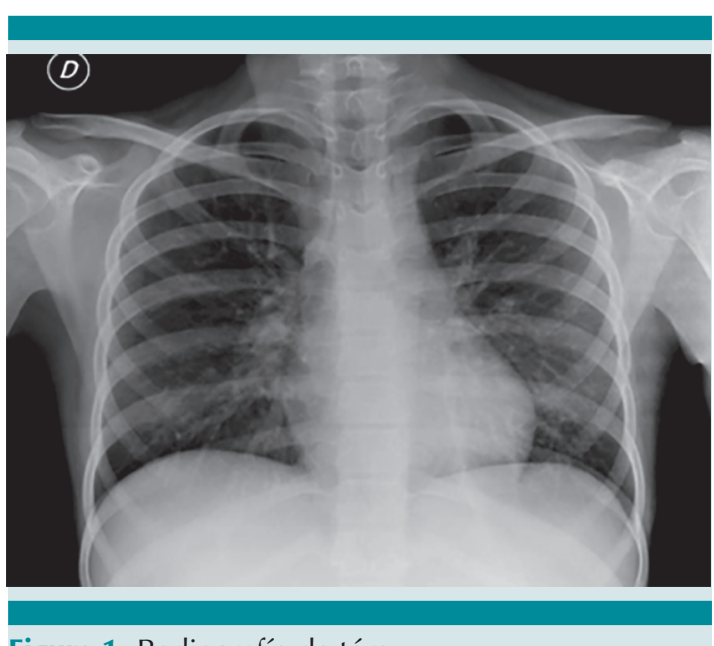

Figura 1. Radiografía de tórax.

intersticial pulmonar, se realizó gasometría arterial en la cual se documenta presión arterial de oxígeno $\left(\mathrm{PaO}_{2}\right) 64 \mathrm{mmHg}$, SPECT/CT con evidencia de concentración del radiotrazador en corteza cerebral, confirmando el diagnóstico de síndrome hepatopulmonar, se programó para realización de EcoC; sin embargo, presentó deterioro clínico con progresión a choque séptico, falla orgánica múltiple y finalmente falleció.

\section{Caso 2}

Niña de 12 años de edad con secuelas de encefalopatía hipóxico isquémica que se presentó por 3 meses de evolución con tos seca y cianosis ungueal, a lo que se agregó disnea progresiva de medianos a mínimos esfuerzos, astenia, adinamia y somnolencia diurna. Clínicamente con oximetría de pulso de $75 \%$, cianosis perioral y ungueal e hipoactividad.

En los exámenes de laboratorio destacó bicitopenia (leucopenia con neutropenia y trombocitipenia). La radiografía de tórax mostró cardiomegalia, rectificación del cono de expulsión de la arteria pulmonar e infiltrado intersticial reticulonodular bilateral, con patrón en vidrio despulido basal derecho (Figura 2). Una semana después presentó insuficiencia respiratoria aguda 


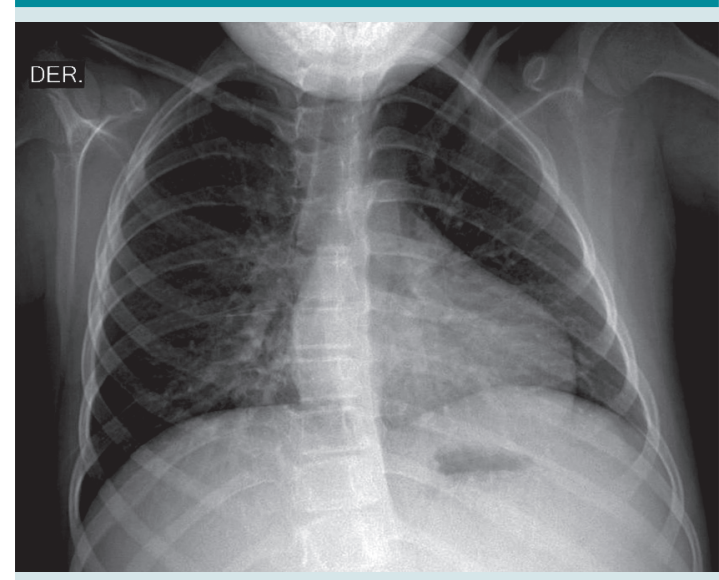

Figura 2. Radiografía de tórax.

para la cual requirió de ventilación mecánica por 11 días, con mejoría clínica con manejo intensivo. Se valoró tomografía computada de alta resolución (TCAR) pulmonar en la que se observó patrón en mosaico bilateral, con áreas de ocupación alveolar e importante aumento del calibre de las estructuras vasculares (Figura 3).

Se realizó EcoTT en el que se describió una PSAP calculada de $22 \mathrm{mmHg}$, con insuficiencia mitral moderada de $38 \mathrm{mmHg}$, adecuada función biventricular, fracción de eyección del ventrículo izquierdo (FEVI) de $72 \%$. Destacando la presencia de un flujo continuo cerca de la vena cava inferior.

Presentó 2 eventos de melena y hematemesis, por lo que se realizó abordaje identificando por panendoscopía várices esofágicas grado III y várices gástricas grado I. Ultrasonido hepático con aumento generalizado de la ecogenicidad, sin lesiones focales. Angio resonancia magnética (AngiolRM) en la que se identificó hipertensión
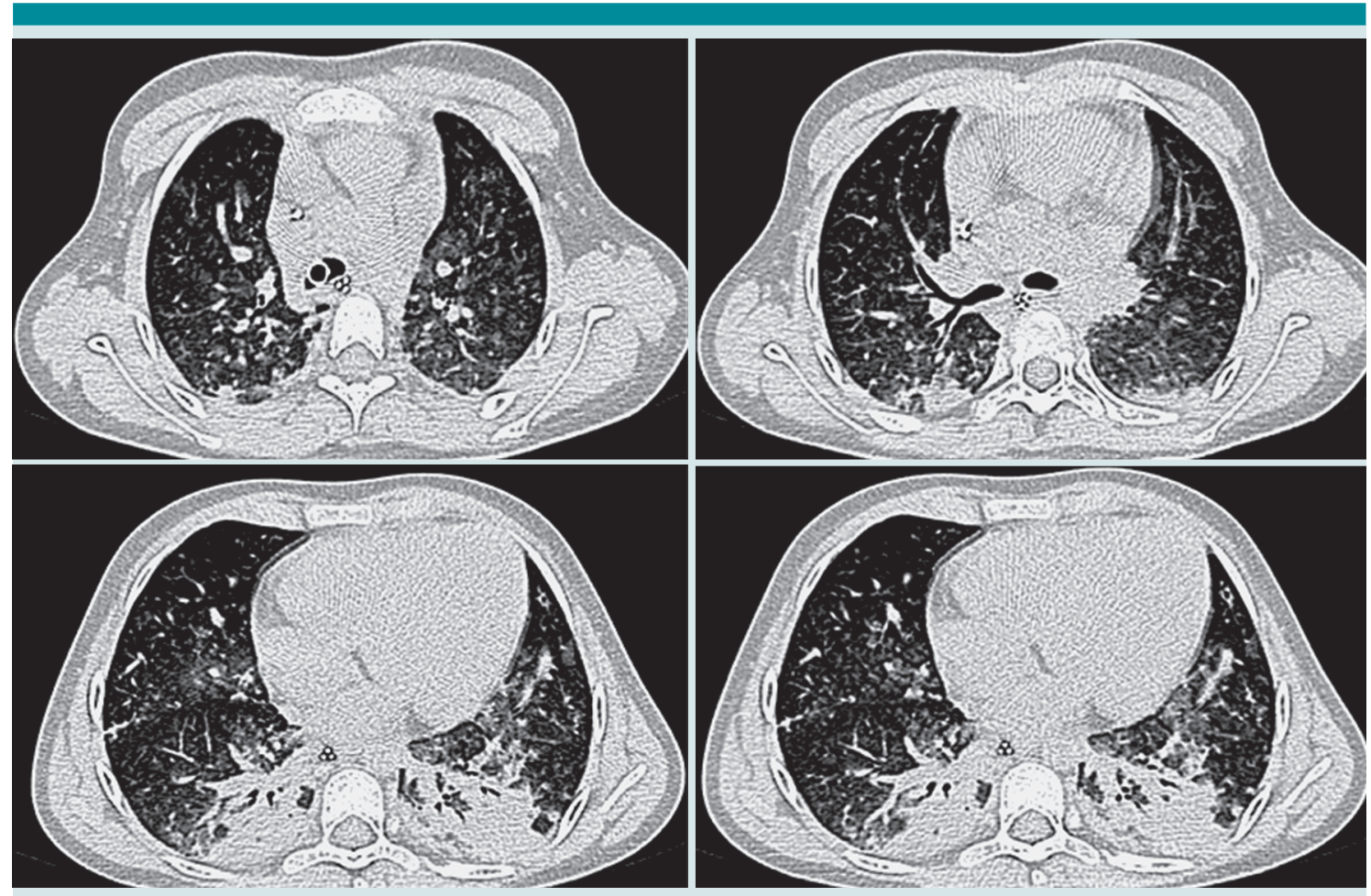

Figura 3. Tomografía computada de alta resolución pulmonar. 
portal con dilatación y tortuosidad del sistema portal, esplenomegalia y vasos colaterales.

Ante la presencia documentada de hepatopatía crónica con hipertensión portal y neumopatía crónica con datos radiológicos y clínicos de afección intersticial, se estableció la sospecha de síndrome hepatopulmonar. En gasometría arterial se reporta $\mathrm{PaO}_{2} 58 \mathrm{mmHg}$.

Se realizó SPECT/CT identificando defectos de concentración del radiotrazador en la periferia de ambos pulmones, con concentración del mismo en bazo, riñones y corteza cerebral, lo cual confirma la presencia de cortocircuito derecha-izquierda. Asimismo, es realizado un EcoC observando paso de contraste de derecha a izquierda después de 3 latidos, sin que se identifiquen alteraciones estructurales cardíacas. De acuerdo con los criterios diagnósticos del síndrome hepatopulmonar se corroboró el diagnóstico y continúo con soporte ventilatorio y realización de derivación esplenorrenal distal (procedimiento de Warren) para manejo de la hipertensión portal.

\section{DISCUSIÓN}

El síndrome hepatopulmonar se define por la triada de hepatopatía, hipoxemia y vasodilatación pulmonar. Su prevalencia global en la población pediátrica es incierta, pero con una morbimortalidad elevada. En pacientes pediátricos el síndrome hepatopulmonar está relacionado con una mortalidad pre trasplante hepático del $25 \%$ al $46 \%{ }^{1,2}$ De acuerdo con algunos estudios enfocados a poblaciones específicas, se estima que del $9 \%$ al $20 \%$ de los pacientes con atresia de vías biliares lo presentan, así como $0.5 \%$ de los pacientes con trombosis de la vena porta. ${ }^{1}$ Otros estudios realizados en centros especializados para trasplante hepático han reportado una prevalencia de entre el $4 \%$ y el $47 \%$; sin embargo, los criterios diagnósticos son variables por lo que no son comparables. ${ }^{3}$
Es una complicación comúnmente asociada a hipertensión portal, no obstante, cualquier hepatopatía crónica y, de cualquier etiología, puede causarla. ${ }^{2}$ La fisiopatología corresponde a un desequilibrio entre la producción y la depuración de sustancias vasoactivas por el hígado, lo que provoca dilatación microvascular dentro de la circulación pulmonar, ocasionando alteración de la ventilación-perfusión mediante un corto circuito funcional y anatómico. ${ }^{4}$

El diagnóstico del síndrome hepatopulmonar se establece demostrando hipoxemia, ya sea por oximetría de pulso o mediante un gradiente alveolo arterial de oxígeno $\left(\mathrm{P} \Delta \mathrm{AaO}_{2}\right) \geq 15 \mathrm{mmHg}$, ambos sin aporte suplementario de oxígeno, la presencia de cortocircuitos intrapulmonares observables por ecocardiografía contrastada (EcoC) o por gammagrama perfusorio con albúmina micro agregada marcada con tecnecio-99 ${ }^{999 m}$ Tc-MAA). ${ }^{1,2,5}$

La positividad del gammagrama para cortocircuitos intrapulmonares se considerará ante la presencia de actividad del radiotrazador en el cerebro o hígado de $\geq 6 \%$. El ecocardiograma, a su vez, será considerado positivo para cortocircuitos intrapulmonares cuando, posterior a la inyección en una vena sistémica de solución fisiológica ( $\mathrm{NaCl} 0.9 \%$ ) agitada, se observan burbujas en la aurícula izquierda después de 3 a 6 ciclos cardíacos. En condiciones de normalidad, estas burbujas serían filtradas en la microcirculación pulmonar, por lo que no serían observables en cavidades izquierdas cardíacas. ${ }^{6}$

Por la naturaleza invasiva de realizar gasometrías arteriales, en pacientes pediátricos se ha aceptado la utilización de la oximetría de pulso como sustituto, siendo $97 \%$ el punto de corte mínimo, lo cual, de acuerdo con Noli et. al. otorga una alta sensibilidad a la prueba. ${ }^{1}$ Sin embargo, un estudio realizado por Hoerning et. al. en Alemania demostraron una pobre relación 
de la oximetría de pulso con el $\mathrm{P} \Delta \mathrm{AaO}_{2}$ para detección temprana de pacientes con síndrome hepatopulmonar en etapas subclínicas (leve a moderada), por lo que una vez que la hipoxemia era aparente por oximetría de pulso la severidad del cuadro era tal que el trasplante hepático era ya un procedimiento de alto riesgo asociado a complicaciones graves, que afectan negativamente a la sobrevida de los pacientes después del mismo. A pesar de esto, se notó que incluso la $\mathrm{P} \Delta \mathrm{AaO}_{2}$ es poco precisa como escrutinio para aquellos pacientes menores de 2 años. ${ }^{2}$

La angiografía, previamente considerada el estándar de oro, es una herramienta para el diagnóstico que ha quedado en desuso en los últimos años, sin embargo, su utilidad es incuestionable. Se identifican 2 patrones angiográficos característicos: el tipo 1 corresponde a un infiltrado fino de aspecto espongiforme en casos de dilataciones mayores y muestra una adecuada reactividad vascular con la suplementación de oxígeno al $100 \%$. El tipo 2 corresponde a comunicaciones arteriovenosas discretas con una pobre reactividad vascular al oxígeno. ${ }^{\text {? }}$

Asimismo, no se requiere de que la enfermedad hepática sea grave; se ha observado su presencia en casos de hepatopatías leves. ${ }^{4}$ El Cuadro 1 muestra las enfermedades hepáticas asociadas al síndrome hepatopulmonar, tanto en población adulta como infantil.

En el contexto general de la enfermedad, es decir, tomando en cuenta no solamente a los niños, sino también a los adultos, la disnea es el principal síntoma y generalmente es insidiosa en su presentación. Asimismo, la desoxigenación arterial en bipedestación u ortodesoxia (disminución de $\geq 5 \%$ o $\geq 4 \mathrm{mmHg}$ de la $\mathrm{PaO}_{2}$ comparada con la posición de supino) es altamente específica en el contexto de hepatopatía crónica. Este fenómeno incrementa el corto circuito funcional debido a que en bipedestación hay una mayor perfusión hacia los segmentos pulmonares basales e inferiores. ${ }^{4}$ La ortodesoxia se suele acompañar de platipnea, definida como incremento de disnea asociado a la posición de bipedestación. ${ }^{3}$

La disnea en pacientes con hepatopatía no se explica únicamente por el síndrome hepatopulmonar, sino que puede estar asociada a otras condiciones como hidrotórax, hipertensión portopulmonar, anemia, ascitis, retención de líquidos o consumo muscular. ${ }^{3}$

La Sociedad Respiratoria Europea (ERS por sus siglas en inglés) clasifica la gravedad del síndrome hepatopulmonar de acuerdo a los valores $\mathrm{P} \Delta \mathrm{AaO}_{2}$ y $\mathrm{PaO}_{2}$ como se muestra en el Cuadro 2. ${ }^{8}$

Cuadro 1. Causas de síndrome hepatopulmonar, no se diferencia entre población pediátrica o adulta, no se enlistan en orden de frecuencia. Modificado de Lange, PA. et. $\mathrm{al}^{4}$

\begin{tabular}{lll}
\hline \multicolumn{2}{l}{ Hepatopatías asociadas al síndrome hepatopulmonar } \\
\hline Cirrosis criptogénica & Deficiencia de $\alpha$-1 Antitripsina & Enfermedad de Gaucher \\
\hline Cirrosis post necrótica & Enfermedad de Wilson & Esquistosomiasis \\
\hline Cirrosis alcohólica & Sarcoidosis & Hiperplasia nodular regenerativa del hígado \\
\hline Cirrosis autoinmune & Hemocromatosis & Rechazo a injerto hepático \\
\hline Cirrosis biliar primaria & Atresia de vías biliares & Histiocitosis de células de Langerhans \\
\hline Hepatitis crónica activa & Hipertensión portal no cirrótica & Síndrome de telómeros cortos \\
\hline $\begin{array}{l}\text { Colangitis esclerosante } \\
\text { primaria }\end{array}$ & Tirosinemia & $\begin{array}{l}\text { Enfermedad de injerto contra huésped hepática secun- } \\
\text { daria a trasplante de precursores hematopoyéticos }\end{array}$
\end{tabular}


Cuadro 2. Modificado de Rodríguez-Roisin, et. $\mathrm{al}^{8}$

\begin{tabular}{|c|c|c|}
\hline Estadio & $\mathrm{P} \triangle \mathrm{AaO}_{2}$ & $\mathrm{PaO}_{2}$ \\
\hline Leve & $\geq 15 \mathrm{mmHg}$ & $\geq 80 \mathrm{mmHg}$ \\
\hline Moderado & $\geq 15 \mathrm{mmHg}$ & $<80 \mathrm{mmHg}$ y $\geq 60 \mathrm{mmHg}$ \\
\hline Grave & $\geq 15 \mathrm{mmHg}$ & $<60 \mathrm{mmHg}$ y $\geq 50 \mathrm{mmHg}$ \\
\hline Muy grave & $\geq 15 \mathrm{mmHg}$ & $\begin{array}{c}<50 \mathrm{mmHg}(<300 \mathrm{mmHg} \\
\left.\text { con } 100 \% \mathrm{FiO}_{2}\right)\end{array}$ \\
\hline
\end{tabular}

La sospecha clínica es fundamental para el diagnóstico oportuno. Síntomas tan sencillos de identificar en los primeros minutos de la consulta como la ortodesoxia y la platipnea se presentan en hasta el $90 \%$ de los pacientes. ${ }^{9}$ Generalmente, la radiografía simple de tórax no muestra alteraciones significativas, por lo que su utilidad radica en la realización de diagnósticos diferenciales.

El síndrome hepatopulmonar se asocia a un mal pronóstico tanto funcional como vital. El retardo en el diagnóstico y, por lo tanto, en la instauración de la terapéutica adecuada se asocia con un pronóstico desfavorable en todos los casos. De acuerdo con Hoerning, et. al. el síndrome hepatopulmonar se describe como una complicación severa en pacientes con cirrosis, por lo que aquellos con enfermedad moderada deben de ser considerados como prioridad para trasplante hepático, debido a que la enfermedad continuará progresando. ${ }^{2}$ Su descripción detallada es de relevancia para incrementar conocimiento médico de la enfermedad y de esta forma poder establecer terapéuticas de forma temprana para mejorar la calidad y esperanza de vida de los pacientes.

El trasplante hepático proporciona una recuperación progresiva de la oxigenación y los datos clínicos característicos como la platipnea y ortodesoxia en más de 85\% de los casos. Asimismo, es el único tratamiento que ha demostrado disminución de los corto circuitos pulmonares evidentes por EcoC. 6,10
Se han investigado alternativas farmacológicas al trasplante hepático, entre las que se incluyen análogos de somatostatina, $\beta$ bloqueadores, inhibidores de ciclooxigenasa, glucocorticoides, inmunosupresores, inhibidores de óxido nítrico, vasoconstrictores pulmonares, óxido nítrico inhalado, antibióticos y ácido gárlico; sin embargo, ninguno de ellos ha demostrado mejoría en la oxigenación y/o en las dilataciones vasculares intrapulmonares. ${ }^{8}$

Existen tratamientos médicos y quirúrgicos que tienen como objetivo el disminuir la progresión del daño hepático y mejorar la sintomatología. La corrección quirúrgica de patología causal del daño hepático, como la cavoplastía en pacientes con síndrome de Budd-Chiari secundario a obstrucción de la vena cava suprahepática o el drenaje de abscesos causales de síndrome de Budd-Chiari, han demostrado reversibilidad del síndrome hepatopulmonar. ${ }^{8}$ Se ha reportado un caso de mejoría de la oxigenación mediante embolización de cortocircuitos intrapulmonares, en un paciente adulto sin mejoría posterior al trasplante hepático, sin embargo, evidencia similar no ha sido demostrada en la población infantil. ${ }^{11}$

El tratamiento inicial posterior al diagnóstico del síndrome hepatopulmonar es sintomático, el cual debe incluir oxígeno suplementario. ${ }^{8}$ Asimismo se debe tratar a la enfermedad hepática de base para detener la progresión de la misma y considerar oportunamente al paciente candidato a trasplante hepático.

Desafortunadamente, en nuestro país las condiciones de pobreza social y la falta de políticas públicas en salud han hecho que el programa de trasplantes no tenga el impacto ni el alcance necesario en la población. Si bien, el trasplante hepático puede ser necesario en pacientes en los que ya se realizó el diagnóstico de síndrome hepatopulmonar. 


\section{CONCLUSIÓN}

La estrategia preventiva del síndrome hepatopulmonar a partir de estrategias de identificación y manejo temprano del paciente con hepatopatía crónica, incluido el trasplante hepático, debe de ser el objetivo primario para evitar la progresión de la enfermedad hepática y la instalación del daño pulmonar.

\section{REFERENCIAS}

1. Noli K, Solomon M, Golding F, Charron M, Ling SC. Prevalence of Hepatopulmonary Syndrome in Children. Pediatrics. 2008;121(3):e522-7.

2. Hoerning A, Raub S, Neudorf $U$, et al. Pulse Oximetry Is Insufficient for Timely Diagnosis of Hepatopulmonary Syndrome in Children with Liver Cirrhosis. J Pediatr. 2014;164(3):1-7.

3. Ho V. Current concepts in the management of hepatopulmonary syndrome. Vasc Health Risk Manag. 2008;4(5):1035-1041.

4. Lange PA, Stoller JK. Hepatopulmonary syndrome: Prevalence, causes, clinical manifestations, and diagnosis. In: Ted W. Post, ed. UpToDate. Waltham, MA: UpToDate; 2016.
5. Erge D, Selimoğlu MA, Karakurt C, et al. Report of seven children with hepatopulmonary syndrome. Turkish J Gastroenterol. 2014;25(6):724-729.

6. Rovira Amigo S, Martín De Vicente C, Bueno Aribayos J, Ortega López J, Girona Comas J, Moreno Galdó A. Síndrome hepatopulmonar en niños: Evaluación y tratamiento. An Pediatr. 2009;71(3):224-229.

7. Carrillo-Esper R, González-Salazar JA, Serralde-Zúñiga A. Síndrome hepatopulmonar. Rev la Fac Med UNAM. 2001;44(5):207-211.

8. Rodríguez-Roisin R, Krowka MJ, Hervé P, et al. Pulmonary-hepatic vascular disorders (PHD). Eur Respir J. 2004;24(5):861-880.

9. Salazar-Marcelino AE, Herrera-García JC, Toledo-Estrada J, Mendoza-Torres MÁ, Romero-Ogawa T. Síndrome hepatopulmonar : Situación clínica en un hospital de tercer nivel en Puebla, México. Neumol Cir Torax. 2016;75(1):18-24.

10. Tumgor G, Arikan C, Yuksekkaya H, et al. Childhood cirrhosis, hepatopulmonary syndrome and liver transplantation. Pediatr Transplant. 2008;12:353-357.

11. Poterucha JJ, Krowka MJ, Dickson ER, Cortese DA, Stanson AW, Krom RAF. Failure of hepatopulmonary syndrome to resolve after liver transplantation and successful treatment with embolotherapy. Hepatology. 1995;21(1):96-100. 